\title{
Pattern Recognition from Compressed Labelled Trees of Fuzzy Regions
}

\author{
Laurent WENDLING ${ }^{1}$, Jacky DESACHY ${ }^{1}$, and Alain PARIES ${ }^{2}$ \\ 1 Université Paul Sabatier (Toulouse III) IRIT 118, route de Narbonne 31062 \\ Toulouse Cedex, France tel: (+33)561.556.599; fax: (+33)561.556.258 \\ E-mail: \{wendling,desachy\}@irit.fr \\ 2 Université Bordeaux I, LaBRI 351, avenue de la Libération 33405 TALENCE \\ CEDEX tel: $(+33) 556.846 .916$; fax: $(+33) 556.846 .669$ \\ E-mail: Alain.Paries@INSAT.fr
}

\begin{abstract}
In this paper, a method of pattern recognition based on images split into a set of trees composed of fuzzy regions is presented. First, a fuzzy segmentation based on possibilistic c-means is carried out in the raster image. Fuzzy support have been defined from a first level cut. On each cluster, a fuzzy region is assumed to be a convex combination of sets with associated features. A set of sample trees is achieved from the application of the segmentation algorithm on characteristic objects. Then, a tree isomorphism to recognize is defined to recognize an object. At last, a new tree compression method is introduced to decrease the complexity when we have to manage with a large set of trees.
\end{abstract}

Keywords: Fuzzy Regions, Tree Isomorphism, Directed Acyclic Graph Compression, Pattern Recognition.

\section{Introduction}

This paper deals with both a new method of pattern recognition based on a tree description of sample images and an optimization approach managing with labelled trees isomorphisms. Currently pattern recognition methods are based on Segmentation - Interpretation cycles which have high processing time costs. The presented approach allows to convert these cycles into a linear application: only one segmentation and one interpretation. First, a fuzzy segmentation based on possibilist c-means is applied on the raster image to obtain fuzzy sets supports. Using fuzzy sets theory, a "convex combination of sets" (also called random sets) is defined, which is composed of a set of crisp regions (defined by successive level-cuts) and a positive weight linked to it. An object is defined by a set of samples (characteristic images) given by an expert. These samples are split with the fuzzy segmentation algorithm into a set of fuzzy regions hierarchical trees. Then, we define the tree isomorphism which links the tree representation of the global image to these sample trees descriptions in order to recognize a particular object. The application complexity increases with the samples set size. So, a new tree compression method is introduced to reduce the processing complexity. 


\section{Pattern Recognition System}

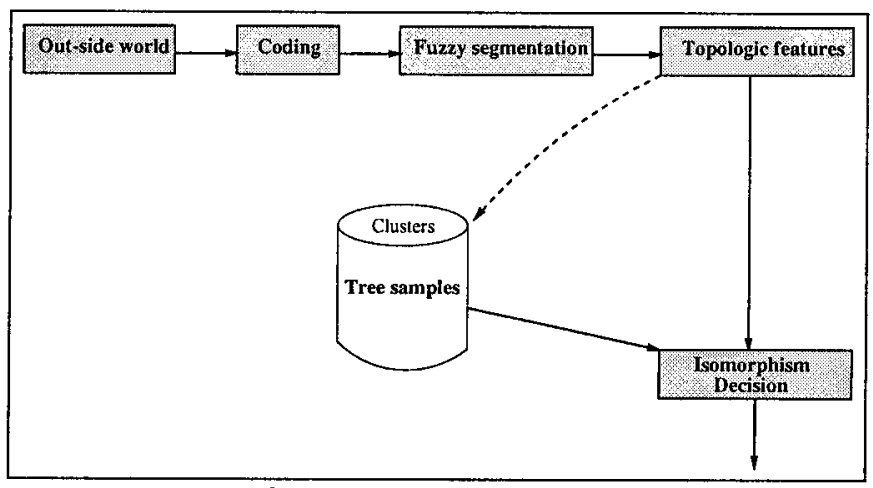

Fig. 1. System Description

The pattern recognition system consists in five parts:

- Fuzzy Segmentation: To split the raster image into a set of fuzzy regions.

- Topological Features: To Compute features associated to each region.

- Tree samples: Each cluster is composed of a set of tree samples.

- Decision part: To Perform a tree isomorphism combined with a similarity ratio in order to find an object with a recognition rate.

\section{$3 \quad$ Fuzzy Segmentation}

\subsection{Fuzzy Partition Definition}

The goal of fuzzy segmentation method is to manage with vagueness boundaries [14] [8] and to allow a pixel to belong more ore less to a given region. Most of fuzzy segmentation methods are based on defining fuzzy partitions by using fuzzy c-means algorithms [3]. Nevertheless these approaches give noisy and non totally coherent results [10] [5]. A recent approach proposed by Krishnapuram [9] seems to overcome these problems. His method is independent of the interclass distance and is based on a "good" membership profile [18]. The initialisation of the defined algorithm is fundamental. Barny \& al. [2] have shown that the use of c-means algorithm to define the input partition can fail by defining coincident clusters. This problem can be overcome if possibilistic c-means algorithm [12] is used with a number of classes equal to 1 . First any partition point is set at 1 . Then the most favourable cluster (validity criteria and partitions variations) is carried out. Then points of cluster data which most verify this new achieved cluster are removed from the image. Processing is runned again until the achievement of unconsistent partitions (too small for example) is performed from the image. Currently clusters validity algorithms have generally high processing times with sometimes unconsistent results [10]. In the present system this algorithm has been applied with a level cut criteria to decrease processing time $(0.9 \mathrm{~s}$. on a $100 \mathrm{MHz}$ SUN SPARC 4). Then a set of partitions composed of fuzzy clusters is achieved. On each cluster fuzzy regions are defined from their support (first level-cut). The hierarchical structure is defined from support inclusions. 


\subsection{Convex Combination of Sets}

Using fuzzy sets theory [17], a fuzzy set can be defined by a convex combination of sets representation [6]. This combination is composed of $n$ included crisp sets $A_{i}\left(A_{1} \supset A_{2} \supset \ldots \supset A_{n}\right)$ with $m\left(A_{i}\right)$ the associated positive weight [15] such as: $\sum_{i=1}^{n} m\left(A_{i}\right)=1$. Assume $n$ values $\left.\left.\alpha_{i} \in\right] 0,1\right]$ and $\alpha_{1}<\alpha_{2}<\cdots<\alpha_{n}$. Let $A=\left\{A_{1}, A_{2}, \cdots, A_{n}\right\}$ be the fuzzy region where $A_{i}$ is the crisp set obtained from the level-cut $\alpha_{i}$. A level-cut, for the value $\alpha_{i}$, is defined as the set of pixels such as membership degree is greater than $\alpha_{i}$. For all crisp set $A_{i}$, the associated weight is (assuming that $\alpha_{0}=0$ ): $\forall A_{i} \subset A, m\left(A_{i}\right)=\alpha_{i}-\alpha_{i-1}$. The membership function of a fuzzy set is obtained from its convex combination of sets representation. This property allows to manage with fuzzy regions by using their level-cuts.

Fuzzy Parameters. A crisp region $A_{i}$ is a set of connected pixels with a nonzero membership degrees value. Features of a region such as perimeter $P$, surface $S$, compactness degree $\frac{S}{P^{2}}$, moments of order one and two... can be computed. The valuation of one particular topologic feature is performed separately on each crisp region by using a measurable function $F$. The final measurable function $\tilde{F}$ of the fuzzy region $A=\left\{A_{1}, A_{2}, \cdots, A_{n}\right\}$, characterising the topologic features, is achieved by the following formula [7]:

$$
\tilde{F}(A)=\sum_{i=1}^{n} m\left(A_{i}\right) \cdot F\left(A_{i}\right)
$$

\section{Tree Isomorphism}

The Euclidian plane of an image can be assumed to the fuzzy sets referential. The first level cut give the support of each fuzzy region. A tree based on the included notion is defined: i.e. let $A$ and $B$ be two fuzzy regions, $s$ and $t$ their corresponding nodes in the tree. $t$ is a successor of $s$ (denoted by $s \rightarrow t$ ) if $B \subset A$ and there is no fuzzy region $C$ such as $B \subset C \subset A$. In fact, fuzzy segmentation splits the image into a hierachical tree.

\subsection{Definitions}

If there exists an arc, in a graph $G$, between $s$ and $t$, then $t$ is a successor of $s$ in $G$. We denote $\operatorname{Succ}_{G}(s)$ the set of all successors of $s$ in $G$. If $t \in S u c c_{G}(s)$, $s$ is the father of $t, s \rightarrow_{G} t$. A graph $G=<V_{G}, M_{G}>$ is defined by the set of its vertice $V_{G}$ and the function $M_{G}$ which, for all $s \in V_{G}$, associates $\operatorname{Succ}_{G}(t)$. $\operatorname{deg}_{G}(s)$ is the degree of $s$, i.e. $\left|\operatorname{Succ}_{G}(s)\right|$. A graph $T$ is a tree iff.:

- There exists a unique vertex, called the root, which is never the ending of an arc. The root of $T$ is denoted by root $T$.

- Each vertex is accessible from the root through a unique path.

- There is no cycle in the underlying undirected graph.

$T / s$ is the sub-tree of $T$ rooted in $s$ and $h e i g h t_{T}(s)$ is the length of the longest finite path starting in $s$. 


\subsection{Isomorphism Search}

Algorithm Description. A depth first search algorithm is applied on the tree image I. Let $s$ be the current node with $\operatorname{deg}_{I}(s)=k, \operatorname{Succ}_{I}(s)=\left\{s_{1}, s_{2}, \ldots, s_{k}\right\}$. For each node $t$, let $\left\{t_{1}, t_{2}, \ldots, t_{k}\right\}$ be its set of successors. Then, a classical isomorphism is used which stores each couple $\{s, t\}$ performed from a tree searched in a list $V$. If a tree of $O_{i}$ is found in the image $I$ tree, a recognition rate is computed. As the number of parameters can be large and as the tree isomorphism has a linear complexity, these two operations can be separeted. This method takes into account images composed of objects set at top level. A basic algorithm [1] which allows to define a tree isomorphism in $\mathcal{O}(n)$ time is used and extended to the present application. Two trees $T_{1}$ and $T_{2}$ are said isomorphic if a tree can be mapped into the other one by permuting the order of the sons of the vertice. The aim of this algorithm is to assign integers to the vertice of the two trees. The processing starts from the leaves and works up towards the roots. Two trees are isomorphic iff. the same integer is assigned to their roots.

Similarity Ratio. A simple mean scheme has been used to carry out the nodes comparison. Other distance measure can obviously be used... Let $s$ be a node of the image tree $I$ and $t$ be a node of the object tree $O_{i}$, with $p$ features:

$$
C(s, t)=\frac{1}{p} \cdot \sum_{i=1}^{p} S\left(P_{i}, P_{i}^{\prime}\right) \text { with: }\left\{\begin{array}{l}
S(0,0)=1 \\
S\left(P_{i}, P_{i}^{\prime}\right)=\frac{\min \left(P_{i}, P_{i}^{\prime}\right)}{\max \left(P_{i}, P_{i}^{\prime}\right)}
\end{array}\right.
$$

A feature can have a greater effect than the others. In this case, a constant $\lambda$ is introduced. Then, the similarity ratio between nodes becomes:

$$
C(s, t)=\frac{1}{\sum_{i=1}^{p} \lambda_{i}} \cdot \sum_{j=1}^{p} S\left(P_{j}, P_{j}\right) \cdot \lambda_{j}
$$

And, for all couples of nodes in $V=\left\{\left\{s^{N 1}, t^{N 1}\right\},\left\{s^{N 2}, t^{N 2}\right\}, \ldots,\left\{s^{N m}, t^{N m}\right\}\right\}$ with $|I / s|=\left|O_{i}\right|=m$ nodes. Assume $\left\{\mu_{1}, \ldots, \mu_{m}\right\}$ is a set of importance degrees with the condition: $\sum_{i=1}^{m} \mu_{i}=1$. Then, an importance degree is assigned to each node as follows:

$$
C \_\operatorname{Tree}\left(I / s, t=O_{i}\right)=\sum_{k=1}^{m} C\left(s^{N k}, t^{N k}\right) \cdot \mu_{k}
$$

The final recognizing rate is: $\operatorname{Reco}\left(I / s, t=O_{i}\right)=100 \cdot \operatorname{C} \_$Tree $\left(I / s, t=O_{i}\right)$

If an expert gives a large number of characteristic sample images for a particular cluster, this application may have a high processing time. So a new labelled tree compression method is introduced to decrease the time complexity. Moreover, this compression allows to perform typical objects (most frequent objects or object parts) and a frequency degree can also be given to each edge in order to apply $A^{*}$ algorithm. 


\section{Tree Compression}

\subsection{Labelling}

Each node of the tree samples can be assumed to be a $\Re^{p}$ value with $p$ the number of topologic parameters associated to the node. The set of all the nodes defines a set of points in a $\Re^{p}$ space. We can suppose that the probability to find two identical $\Re^{p}$ points is very low, but as the expert gives representation of a typical object, the probability to find two nearest $\Re^{p}$ points is high. The idea is to compute the clusters defined by these sets of points. An unsupervised method must be used because, at the begining, the number of clusters is unknown. We have also used the algorithm presented subsection 3.1. A label corresponding to a cluster center is assigned to each cluster. A set of labelled trees $\left\{T_{1}, T_{2}, \ldots, T_{k}\right\}$ is achieved. At last, this set of trees is grouped into a single tree $T$ such as: $\operatorname{Succ}_{T}\left(\operatorname{root}_{T}\right)=\left\{\operatorname{root}_{T_{1}}, \operatorname{root}_{T_{2}}, \ldots, \operatorname{root}_{T_{k}}\right\}$ and Label $\left(\operatorname{root}_{T}\right)=0$ [16].

\subsection{Directed Acyclic Graph Compression}

Let $G=<V_{G}, M_{G}>$ be a DAG (i.e. a Directed Acyclic Graph) and $s \in V_{G}$. Tree $(G, s)$ is the tree of all finite paths starting from s. If $T=\left\langle V_{T}, M_{T}\right\rangle$ is a labelled tree, Label $(t)$ is noted as the label of the node $t \in V_{T}$. As described in [4], a unique DAG can be built: $G=\operatorname{Dag}(T)$ such as $T$ and $\operatorname{Tree}\left(G\right.$, root $\left._{G}\right)$ are isomorphic. In this paper, this approach is extended to labelled tree. An equivalence relation $\approx_{L}$ is defined such as: $\forall t, s \in V_{T}, t \approx_{L} s$, iff. $T / t$ and $T / s$ are isomorphic and $\operatorname{Label}(t)=\operatorname{Label}(s)$. So, for all tree $T=\left\langle V_{T}, M_{T}\right\rangle$, $\operatorname{Dag}(T)$ is the graph $\left\langle V^{\prime}, M^{\prime}\right\rangle$ such as:

$-V^{\prime}=V / \approx_{L}$

$-M^{\prime}([s])=\left\{\left[s_{1}\right], \ldots,\left[s_{k}\right]\right\}$ with $\left[s_{i}\right]$ the cluster number of $s_{i}, \operatorname{Succ}_{T}(s)=$ $\left\{s_{1}, \ldots, s_{k}\right\}$ and $\operatorname{Label}\left(\left[s_{i}\right]\right)=\operatorname{Label}\left(s_{i}\right)$.

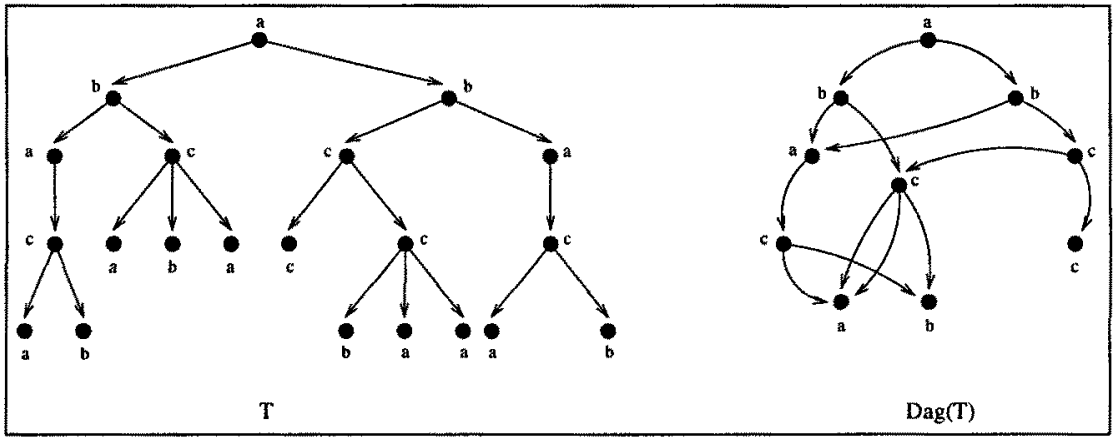

Fig. 2. DAG compression (example)

For all tree $T=\left\langle V_{T}, M_{T}>, \operatorname{Dag}(T)\right.$ can be built in $\mathcal{O}(n)$ with $\left|V_{T}\right|=n$. Let $T=<V_{T}, S_{T}>$ be a tree. $D=\left\langle V_{D}, S_{D}\right\rangle$ is going to be built with $V_{D}=\{1,2, \ldots, n\}$, and $p: V_{T} \rightarrow V_{D}$ such as $p(v)$ is the element of $V_{D}$ which 
represents the equivalence cluster of $v$ for $\approx_{L} . S_{D}$ is such that $S_{D}(p(v))=$ $\left\{p\left(v_{1}\right), p\left(v_{2}\right), \ldots, p\left(v_{k}\right)\right\}$ with $S_{T}(v)=\left\{v_{1}, v_{2}, \ldots, v_{k}\right\}$. For all $v, v^{\prime} \in V_{T}, v \approx_{L}$ $v^{\prime} \Rightarrow$ height $_{T}(v)=$ height $_{T}\left(v^{\prime}\right)$. Let $h \in N, B_{h}=\left\{v / v \in V_{T}\right.$, height $\left.(v)=h\right\}$ is defined. For all $v, v^{\prime} \in B_{h}: v \approx_{L} v^{\prime} \leftrightarrow S_{D}(v)=S_{D}\left(v^{\prime}\right)$ and $\operatorname{Label}(v)=$ Label $\left(v^{\prime}\right)$. For all $w \in V_{T}$, the serie $s(w)$ is associated to the integer representing $S_{D}(p(w))$, in increasing order. On every set $B_{h}$, a global order $v \prec$ $v^{\prime} \Leftrightarrow p\left(S_{T}(v)\right) \leq_{l e x} p\left(S_{T}\left(v^{\prime}\right)\right)$ is computed. $\leq_{l e x}$ is the lexicographical order on the vertex series of $T$ associated wih $\leq . v \approx_{L} v^{\prime} \Leftrightarrow v \prec v^{\prime}, v^{\prime} \prec v$ and $\operatorname{Label}(v)=\operatorname{Label}\left(v^{\prime}\right)$. On the set of labels, the global order $<_{L}$ is defined.

\section{Achieved Results and Discussion}

\subsection{Elliptical Biologic Cells Recognition}

- Elliptical Cells Cluster. These little images (30 in the present application) are selected on cells images and represent prototypes to find.

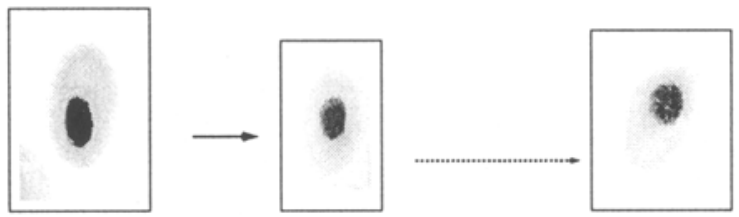

- Fuzzy Segmentation. A fuzzy segmentation is carried out from possibilistic c-means algorithm (subsection 3.1). Compactness and ellipticity degree (based on moments form 0 to 2 [13]), features are computed for each fuzzy region. A 0.75 weight is, a priori, attributed to the cytoplasm node, and a 0.25 weight is attributed to the kernel node because the main information (elliptical cell) is given by the cytoplasm.

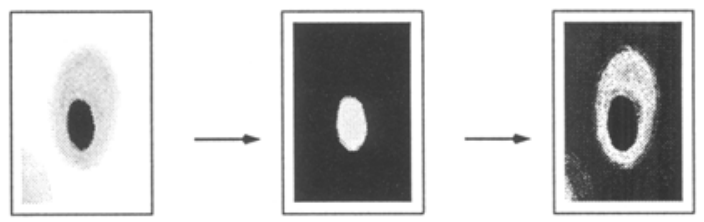

- Application. A test image composed of typical cells which are usally found in cells images is defined. Cells which reach at least a $90 \%$ recognition rate are recognized (the following images represent the basic image and its fuzzy partition). After applying the isomorphism algorithm, we achieve there is two elliptical cells with a $94.37 \%$, for the best one, recognition rate in the image.

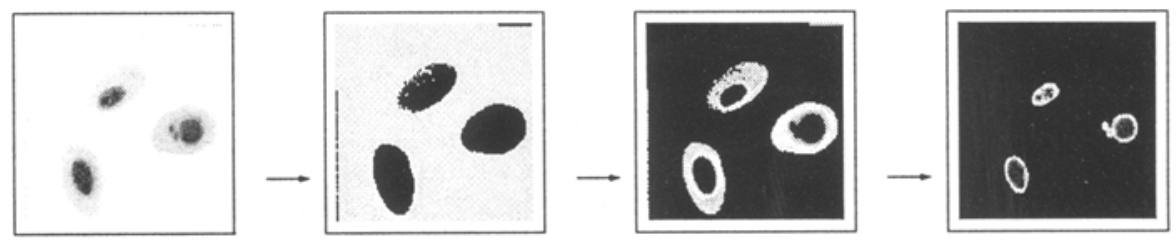




\subsection{Second Application of the Method: Mice Detection}

- Mice Cluster. A set of characteristic mice (catched in the lab) is defined.

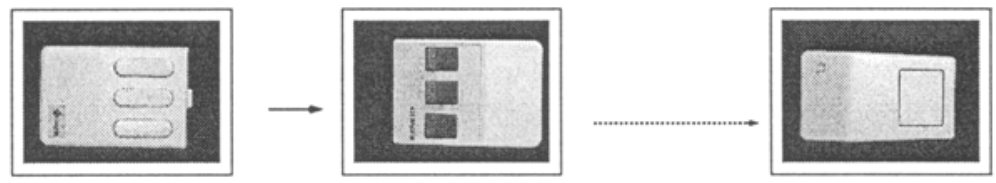

- Fuzzy Segmentation. A fuzzy segmentation is achieved. For each fuzzy region compactness feature is computed (image and its fuzzy partition).

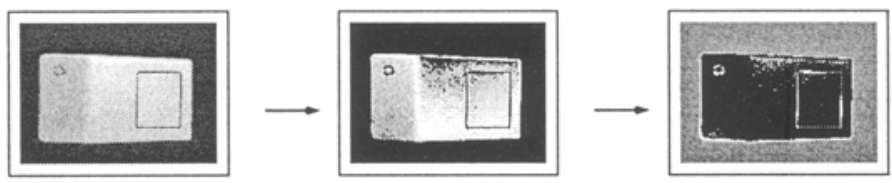

\section{- Test Image.}

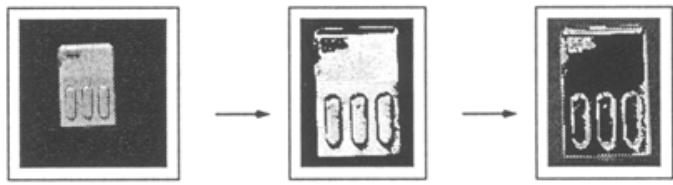

After searching the tree isomorphism, the associated similarity degree is computed. A little default (upper left corner of the test mouse) is found in the test image (maximum recognition rate is $94 \%$ for the sample type number one).

\subsection{Remarks}

A compression rate around $75 \%$ is reached for the previous applications. Results have been achieved 3.5 times faster due to the compression method. These examples are too simple to show the power of the proposed compression approach. Currently, studies are in progress with more complex scenes. Nevertheless, 4 sets $A_{1}, A_{2}, A_{3}$ and $A_{4}$ of random trees have been generated using a well known one-to-one correspondance between Dyck language and trees. Each set contains 100 trees of size 50 for $A_{1}, 60$ for $A_{2}, 100$ for $A_{3}$, and 200 for $A_{4}$. A comparison of compression rates can be found on the table bellow:

\begin{tabular}{||c|c|c|c|c|c|}
\hline Nb. of labels & 1 & 2 & 3 & $\ldots$ & 10 \\
\hline$A_{1}$ & $62 \%$ & $53 \%$ & $49 \%$ & $\ldots$ & $34 \%$ \\
\hline$A_{2}$ & $64 \%$ & $54 \%$ & $49 \%$ & $\ldots$ & $35 \%$ \\
\hline$A_{3}$ & $67 \%$ & $59 \%$ & $54 \%$ & $\ldots$ & $43 \%$ \\
\hline$A_{4}$ & $71 \%$ & $63 \%$ & $59 \%$ & $\ldots$ & $47 \%$ \\
\hline
\end{tabular}

This table does not take into account objects likeness. If an expert gives several objects belonging to the same cluster, the compression rate would be higher. A frequency degree can be defined as follows: $\frac{\text { duplicate edges }}{\text { total number of edges }}$. 


\section{Conclusion}

A new method of pattern recognition based on a tree description of sample images from a fuzzy segmentation method and an optimization method for managing labelled trees isomorphisms (isomorphism and labelled tree compression) has been introduced. This method has been successfully applied on different kinds of application. Currently, we try to generalize this method to more complex scenes with graphs ismorphisms involving relations between nodes like distance between two fuzzy regions, orientation degree between two fuzzy regions...

\section{References}

1. A.V. Aho, J. E. Hopcroft and J. D. Ullmann, "The Design and Analysis of Computer Algorithms", book, ed. Addison Wesley, 1974.

2. M. Barni, V. Cappellini and A. Mecocci, Comments on "A Possibilistic Approach to Clustering", IEEE Transactions on Fuzzy Systems, vol 4:3, 1996, pp 393-396.

3. J.C. Bezdek, Pattern Recognition with Fuzzy Objective Function Algorithms, Plenum Press, New York, 1981.

4. B. Courcelle and A. Pariès, "Mineur d'arbres avec racines", Informatique Théorique et Applications, 1995.

5. R.N. Dave, Characterization and Detection of Noise in Clustering, Pattern Recognition Letters, vol 12, 1991, pp 657-664.

6. D. Dubois and M.C. Jaulent, "A General Approach to Parameter Evaluation in Fuzzy Digital Pictures", Pattern Recognition Letters 4, 1987, pp 251-261.

7. D. Dubois and H. Prade, "Possibility Theory, an Approach to the Computerized Processing of Uncertainty", Plenum Press, New-York, 1988.

8. J.M. Keller and C. L. Carpenter, "Image Segmentation in the Presence of Uncertainty", International Journal of Intelligent Systems, Vol. 5, Iss 2, 1990, pp. 193-208.

9. Raghu Krishnapuram, "Fuzzy Clustering Methods in Computer Vision", EUFIT'93, Aachen, Sep. 7, 1993, pp 720-730.

10. R. Krishnapuram, "Generation of Membership Functions via Possibilistic Clustering", $3^{\text {rd }}$ IEEE Conference on Fuzzy System, vol 3, 1994, pp 902-908.

11. R. Krishnapuram and J.M. Keller, "A Possibilistic Approach to Clustering", IEEE Transactions on Fuzzy Systems, vol 1:2, 1993, pp 98-110.

12. R. Krishnapuram and J.M. Keller, "The Possibilistic C-Means Algorithm: Insights and Recommendations", IEEE Transactions on Fuzzy Systems, vol 4:3, 1996, pp 385-393.

13. Reed Teague, "Image Analysis via the General Theory of Moments", J. Optical Society of America, Vol. 70, N. 8, 1980, pp 920-921.

14. E.H. Ruspini, "A New Approach to Clustering", Inform. and Control, vol 15, 1969, pp 22-32.

15. G. Shafer, "A Mathematical Theory of Evidence", Princetown University Press, 1976.

16. L. Wendling, J. Desachy and A. Paries "Pattern Recognition by Splitting Images Into Trees of Fuzzy Regions", International Journal of Intelligent Data Analysis, Elsevier, 1997.

17. L. A. Zadeh, "Fuzzy Sets", Information and Control 8, pp 338-353, 1965.

18. H. J. Zimmerman and P. Zysno, "Quantifying Vagueness in Decision Models", European J. Operational Res., vol 22, 1985, pp 148-158. 